\title{
Zoom systems with tuneable lenses and linear lens movements
}

\author{
Leonhard Lenk ${ }^{*}$, Beate Mitschunas and Stefan Sinzinger
}

\begin{abstract}
Background: Classical zoom lenses are based on movements of sub-modules along the optical axis. Generally, a constant image plane position requires at least one nonlinear sub-module movement. This nonlinearity poses a challenge for the mechanical implementation.

Tuneable lenses can change their focal length without moving along the optical axis. This offers the possibility of small system lengths.

Since the focal range of tuneable lenses with significant aperture diameters is still limited, the use of tuneable optics in zoom lenses is usually restricted to miniaturized applications.

Methods: To solve the challenge of the nonlinear movement in classical zoom lenses and the limitations of tuneable lenses for macroscopic applications we propose a combination of both concepts. The resulting 'Hybrid Zoom Lens' involves linear movements of sub modules as well as changing the focal length of a tuneable lens.

The movements of the sub-modules and the focal length tuning of the lens are already determined by the collinear layout of the zoom lens. Therefore, we focus on collinear considerations and develop a method that allows a targeted choice of specific collinear layouts for our 'Hybrid zoom lenses'.

Results: Based on examples and an experimental setup we demonstrate the feasibility of our approach. We apply the proposed method to examples of classical zoom lenses and zoom lenses based exclusively on tuneable lenses. Thereby we are able to show possible advantages of our 'Hybrid zoom lenses' over these widespread system types.

Conclusions: We demonstrate important collinear considerations for the integration of tuneable lenses into a zoom lens. We show that the combination of classical zoom lens concepts with tuneable lenses offers the possibility to reach smaller system lengths for macroscopic zoom lenses while requiring only a small focal tuning range of the tuneable lens.
\end{abstract}

Keywords: Zoom lens, Optics design, Tuneable lens, Collinear

\section{Introduction}

The main task of a photographic zoom lens is the variation of the overall focal length while keeping the image plane at a constant position. In classical zoom lenses this is achieved by moving at least two sub modules. There are two basic operating principles of these classical zoom lenses, described e.g. in [1]: The so-called opto-mechanical compensation requires only linear movements of the sub-modules, but does not achieve a constant image plane position over the whole zoom range. The so-called mechanical compensation involves two different types of moving sub-modules (Fig. 1): The variator usually moves linearly along the

\footnotetext{
* Correspondence: leonhard.lenk@tu-ilmenau.de

TU IImenau, Institut für Mikro- und Nanotechnologien - IMN MacroNano ${ }^{\oplus}$, Fachgebiet Technische Optik, 98693 IImenau, Germany
}

optical axis to change the systems focal length. The compensator moves along the optical axis to compensate the image plane dislocation caused by the variator's movement. For this task the trajectory of the compensator always has to be nonlinear. The mechanical implementation of this trajectory requires complex gear systems. We analyse the potential to optimize the configuration of the optical system by applying tuneable optical elements.

Tuneable lenses offer the possibility of changing their focal length without elements moving along the optical axis (e.g. [2] [3] [4] [5]). Therefore, no additional installation space is needed to allow for such a longitudinal movement. This results in a potential reduction of the size and weight of optical systems by using tuneable lenses (e.g. [6] [7]). Apart from that, many tuneable lenses can be 


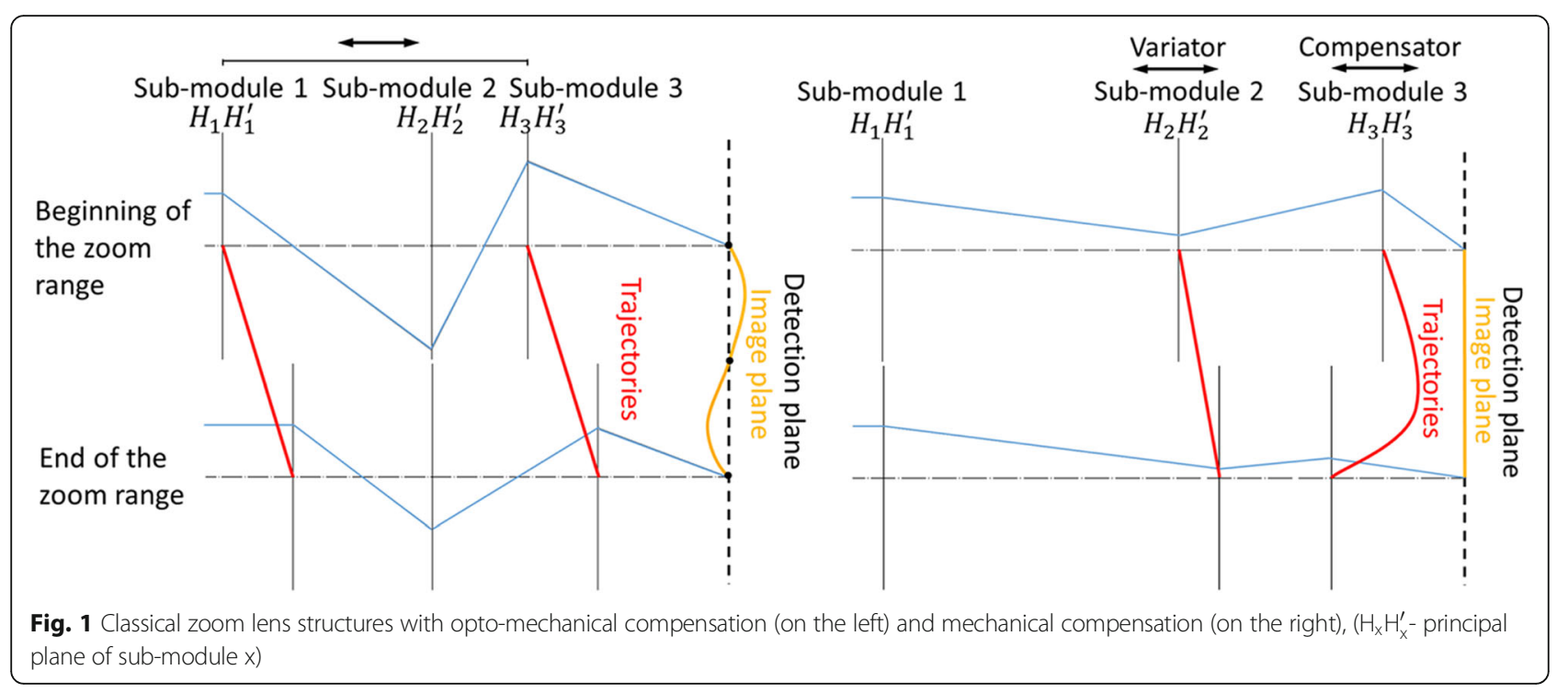

controlled electrically. Therefore, it is easier to calibrate the focal length change of a tuneable lens compared to the trajectory of a sub-module realized by the mechanical gear systems generally used for classical zoom lenses.

The choice and focal range of commercially available tuneable lenses with aperture diameters of at least $10 \mathrm{~mm}$ is quite limited. This also limits the possible zoom range of a zoom system based on tuneable lenses. In miniaturized systems, as used e.g. in smartphone cameras, this problem can be solved by using multiple zoom lenses and dividing the zoom range of the overall system. This is common practice without tuneable lenses [8]. The method seems impractical in macroscopic applications like photographic zoom lenses considering their larger lens diameters. This may be one reason why the application of tuneable lenses in zoom systems is currently restricted to miniaturized systems (e.g. [9]).

Considering the disadvantages of classical zoom lenses and the limitations of tuneable lenses in macroscopic applications we propose a combination of both concepts to find an optimum configuration. The aim is to analyse the possibilities of realizing macroscopic 'Hybrid Zoom Lenses' with solely linearly moving sub modules and a constant image plane.

Since the trajectories of the sub-modules as well as the focal length change of the tuneable lens are already determined by the collinear layout of the zoom system, we focus on the optimization of the collinear design. We restrict our considerations to photographic zoom lenses consisting of three sub-modules. We also require a constant distance between the first sub-module and the image plane, which offers the possibility of using the first sub-module for focusing.

In the next section we present the steps of our approach to the presented topic, by giving an overview of the possible hybrid zoom lens structures. After that we derive an approach for reaching a specific collinear layout for a chosen structure. We demonstrate a method that allows us to systematically analyse possible solutions and to make a targeted choice of a specific collinear layout that meets the chosen requirements. We present an experimental setup that demonstrates the feasibility of our theoretical concepts. In the final sections we discuss possible advantages as well as challenges of our hybrid photographic zoom lenses.

\section{Methods}

\section{Determination of the system's layout}

In spite of numerous restrictions there are still many possibilities for integrating a tuneable lens into a classical zoom lens structure. Table 1 compares the various options for a layout of the 'Hybrid Zoom Lens' system (Table 1).

We choose system variation number four for further consideration since it seems most promising for reducing the overall system complexity. When using this variation only the second sub-module is moving. Simultaneously it changes its own focal length to keep the image plane position constant. Thereby it fulfils the tasks of both the classical variator and the compensator. This already determines the following general collinear layout (Fig. 2).

Table 1 Possible hybrid zoom lens structures (t-tuneable, mmoving)

\begin{tabular}{|c|c|c|c|}
\hline Variation No. & Sub-module 1 & Sub-module 2 & Sub-module 3 \\
\hline $\mathbf{1}$ & $\mathrm{t}$ & $\mathrm{m}$ & \\
\hline $\mathbf{2}$ & $\mathrm{t}$ & $\mathrm{m}$ & $\mathrm{m}$ \\
\hline $\mathbf{3}$ & $\mathrm{t}$ & $\mathrm{m}$ & $\mathrm{m}$ \\
\hline $\mathbf{4}$ & & $\mathrm{t}$ and $\mathrm{m}$ & \\
\hline $\mathbf{5}$ & & $\mathrm{t}$ & $\mathrm{m}$ \\
\hline $\mathbf{6}$ & & $\mathrm{t}$ and $\mathrm{m}$ & $\mathrm{m}$ \\
\hline $\mathbf{7}$ & & & $\mathrm{t}$ \\
\hline $\mathbf{8}$ & & $\mathrm{m}$ & $\mathrm{t}$ and $\mathrm{m}$ \\
\hline $\mathbf{9}$ & & \multicolumn{3}{|}{} \\
\hline
\end{tabular}




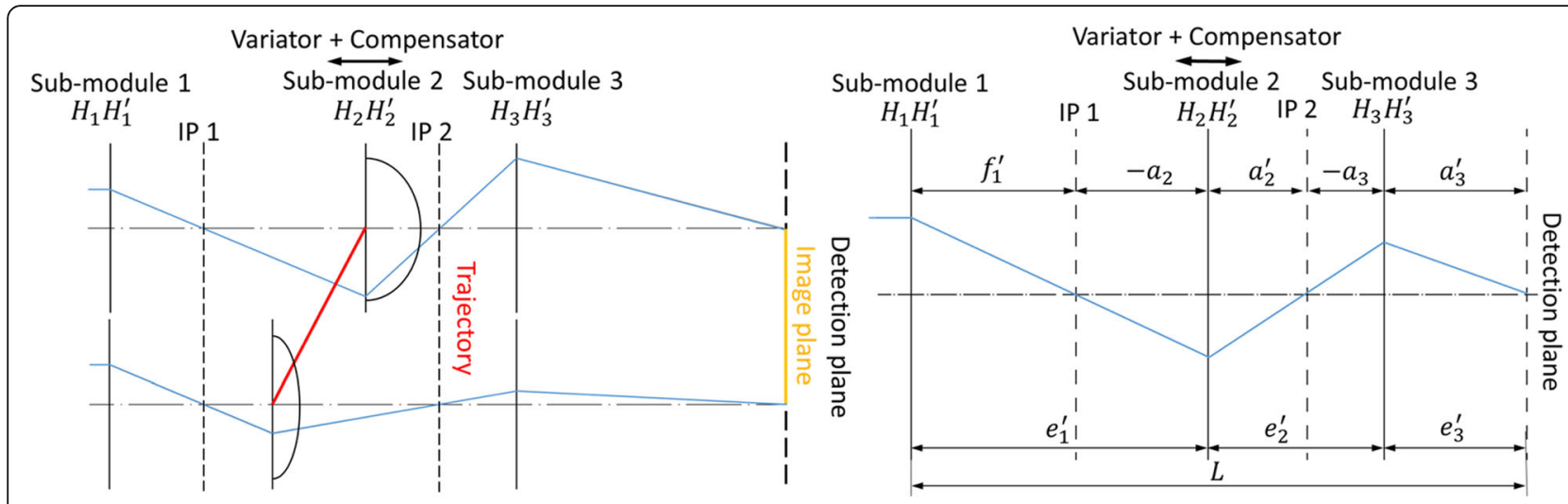

Fig. 2 Structure and general collinear layout of the proposed hybrid zoom lens (IP-intermediate image plane)

\section{System specifications}

When starting to design a new zoom lens for photographic applications there are usually some fixed requirements, such as a specific overall focal length at the beginning of the zoom range $f_{A}^{\prime}$, and a zoom ratio $V=\frac{f_{E}^{\prime}}{f_{A}^{\prime}}$ . The indices " $A$ " and " $E$ " here mean "at the beginning" and "at the end" of the tuning range of the overall focal length respectively. Often there are also some fixed requirements concerning a maximum installation space such as a maximum system length $L_{\max }$.

As can be seen from the general collinear layout shown above (Fig. 2), it is necessary to derive many system parameters from a small set of fixed constraints. Therefore, we need additional input parameters for our calculations in order to end up with a specific collinear layout.

At this point, it makes sense to think about possible additional constraints before setting up equations: Since we have to consider the system performance over the whole zoom range, system parameters that do not change over the focal range will reduce the degree of freedom most efficiently. Therefore, we choose the two constant focal lengths of the nonmoving sub-modules $f_{1}^{\prime}$ and $f_{3}^{\prime}$ as well as the system length $L$.

\section{Geometrical relations}

The next step is to derive equations for all of the unknown system parameters depending only on the fixed requirements and the chosen additional constraints. To this end, we take a look at the general collinear layout (Fig. 2). Here we apply the usual sign convention where all distances are counted as positive values in the direction of light propagation, starting from the respective principal plane, and as negative if they go against the direction of light propagation. Using the basic optical equation

$$
\beta^{\prime}=\frac{f^{\prime}}{a+f^{\prime}}
$$

Where " $\beta$ " " denotes the magnification of a system with focal length " $f$ " and distance "a" between the principal plane and the object. Thus, we can derive some useful geometrical relations (Fig. 2):

$$
\begin{aligned}
& e_{1}^{\prime}=f_{1}^{\prime}+f_{2}^{\prime}\left(1-\frac{1}{\beta_{2}^{\prime}}\right) \\
& e_{3}^{\prime}=a_{3}^{\prime}=f_{3}^{\prime}\left(1-\beta_{3}^{\prime}\right)=\text { constant } \\
& e_{2}^{\prime}=L-e_{1}^{\prime}-a_{3}^{\prime}
\end{aligned}
$$

$$
L=e_{1}^{\prime}+a_{2}^{\prime}-a_{3}+a_{3}^{\prime}
$$

Here " $f_{1}^{\prime}$ "denotes the focal length of sub-system number one, " $\beta_{2}^{\prime}$ "the magnification of sub-module number two and so on. We now use the well-known relation

$$
\beta^{\prime}=\frac{a^{\prime}}{a}
$$

where " $a$ ' " denotes the distance from principal plane to the image. In a general system, we are thus able to eliminate $a_{2}^{\prime}$ and $a_{3}$ from eq. (5):

$$
L=e_{1}^{\prime}+\beta_{2}^{\prime}\left(f_{1}^{\prime}-e_{1}^{\prime}\right)+a_{3}^{\prime}\left(-\frac{1}{\beta_{3}^{\prime}}+1\right)
$$

The overall focal length of the system $f$ can be described by the following expression:

$$
f^{\prime}=f_{1}^{\prime} \beta_{2}^{\prime} \beta_{3}^{\prime}
$$

Using (8) to express $\beta_{3}^{\prime}$ within (7) and applying (2) and (3) we can set up the following quadratic equation: 
$L=\beta_{2}^{\prime}\left(-f_{2}^{\prime}-\frac{f_{1}^{\prime} f_{3}^{\prime}}{f^{\prime}}\right)+f_{1}^{\prime}+2 f_{2}^{\prime}+2 f_{3}^{\prime}+\frac{1}{\beta_{2}^{\prime}}\left(-f_{2}^{\prime}-\frac{f_{3}^{\prime} f^{\prime}}{f_{1}^{\prime}}\right)$

The solutions of this equation are:

$$
\begin{aligned}
& \beta_{2-1,2}^{\prime}=\frac{-y \pm \sqrt{y^{2}-4 x z}}{2 x} \\
& x=-f_{2}^{\prime}-\frac{f_{1}^{\prime} f_{3}^{\prime}}{f^{\prime}} \\
& y=f_{1}^{\prime}+2 f_{2}^{\prime}+2 f_{3}^{\prime} \\
& z=-f_{2}^{\prime}-\frac{f_{3}^{\prime} f^{\prime}}{f_{1}^{\prime}}
\end{aligned}
$$

\section{Petzval sum as additional boundary condition}

Equation (10) gives us the possibility to calculate two solutions for $\beta_{2}^{\prime}$ in a position where we know both, $f$, which is true for the beginning and the end of the zoom range, and the focal length $f_{2}^{\prime}$ of the tuneable sub-module. We thus need an additional condition for the determination of the focal length of the tuneable sub-module at the end or the beginning of the zoom range. For this we use the well-known Petzval condition:

$$
\frac{F_{1}}{n_{1}}+\frac{F_{2}}{n_{2}}+\frac{F_{3}}{n_{3}}=0
$$

Where $F_{1}, F_{2}$ and $F_{3}$ are the refractive powers of the three sub modules and $\mathrm{n}_{1}, \mathrm{n}_{2}$ and $\mathrm{n}_{3}$ are their refractive indices.

At this stage we do not know the refractive indices of the sub-modules yet, which will most likely consist of many lenses with different refractive indices; we assume that the sub modules have the same refractive indices. This will not be too far from the real solutions since the differences of the refractive indices will be far smaller than the differences of the refractive powers $F_{1}$ to $F_{3}$. When using the focal lengths instead of the refractive powers we get:

$$
0=\frac{1}{f_{1}^{\prime}}+\frac{1}{f_{2}^{\prime}}+\frac{1}{f_{3}^{\prime}}
$$

The varying focal length of the tuneable submodule $f_{2}^{\prime}$ does not allow us to fulfil condition (12) across the whole zoom range. Since we define the beginning of the zoom range as the position with the smallest overall focal length, this will be also the position with the biggest object field angle. This also means that the beginning of the zoom range will be the position most affected by field curvature, which is reduced by fulfilling the Petzval condition. Therefore, it makes sense to fulfil the Petzval condition at the beginning of the zoom range and use it to calculate the focal length of the tuneable sub-module in this position.

With this additional boundary condition we are now able to calculate all system parameters at the beginning of the zoom range. It gives us all system parameters which stay constant over the zoom range. We now need to calculate values for the system parameters that change while zooming. This needs to be performed over the whole zoom range. The most important parameters are $e_{1}^{\prime}$ and $f_{2}^{\prime}$.

Since $a_{3}^{\prime}, \beta_{3}^{\prime}$ and $f_{1}^{\prime}$ stay constant over the zoom range, we can use (8) to calculate $\beta_{2}^{\prime}$ at any given overall focal length $f$ within the zoom range. We use (5), (6) and (2) to receive the missing values of $e_{1}^{\prime}$ and $f_{2}^{\prime}$ :

$$
\begin{aligned}
& e_{1}^{\prime}=f_{1}^{\prime}-\frac{f_{1}^{\prime}+a_{3}^{\prime}\left(1-\frac{1}{\beta_{3}^{\prime}}\right)-L}{1-\beta_{2}^{\prime}} \\
& f_{2}^{\prime}=\frac{e_{1}^{\prime}-f_{1}^{\prime}}{1-\frac{1}{\beta_{2}^{\prime}}}
\end{aligned}
$$

\section{Algorithm for finding the appropriate systems layout}

The calculation approach presented above requires the following steps and leads to two results (Fig. 3):

\section{Automated variation of parameters}

The steps above show that we have to choose values for several input parameters in advance, without knowing if any of the two resulting collinear layouts will be feasible. The most important criteria for the feasibility of the proposed zoom lens are the distances between the sub-modules. These generally have to be positive values over the whole zoom range in order to avoid collisions. Another important factor is the focal length of the second sub-module $f_{2}^{\prime}$, which has to stay within the focal range of the tuneable lens. This means that we have to apply an iterative approach: After following the various steps to define the systems layout we have to control the system's properties mentioned above. The range of possible values for the additional input parameters usually is too large to get an overview of the feasible solutions by just performing the iterations manually. There would be no possibility of a targeted choice of a specific collinear layout. Therefore, it is desirable to automate the variation of parameters as well as to control and 


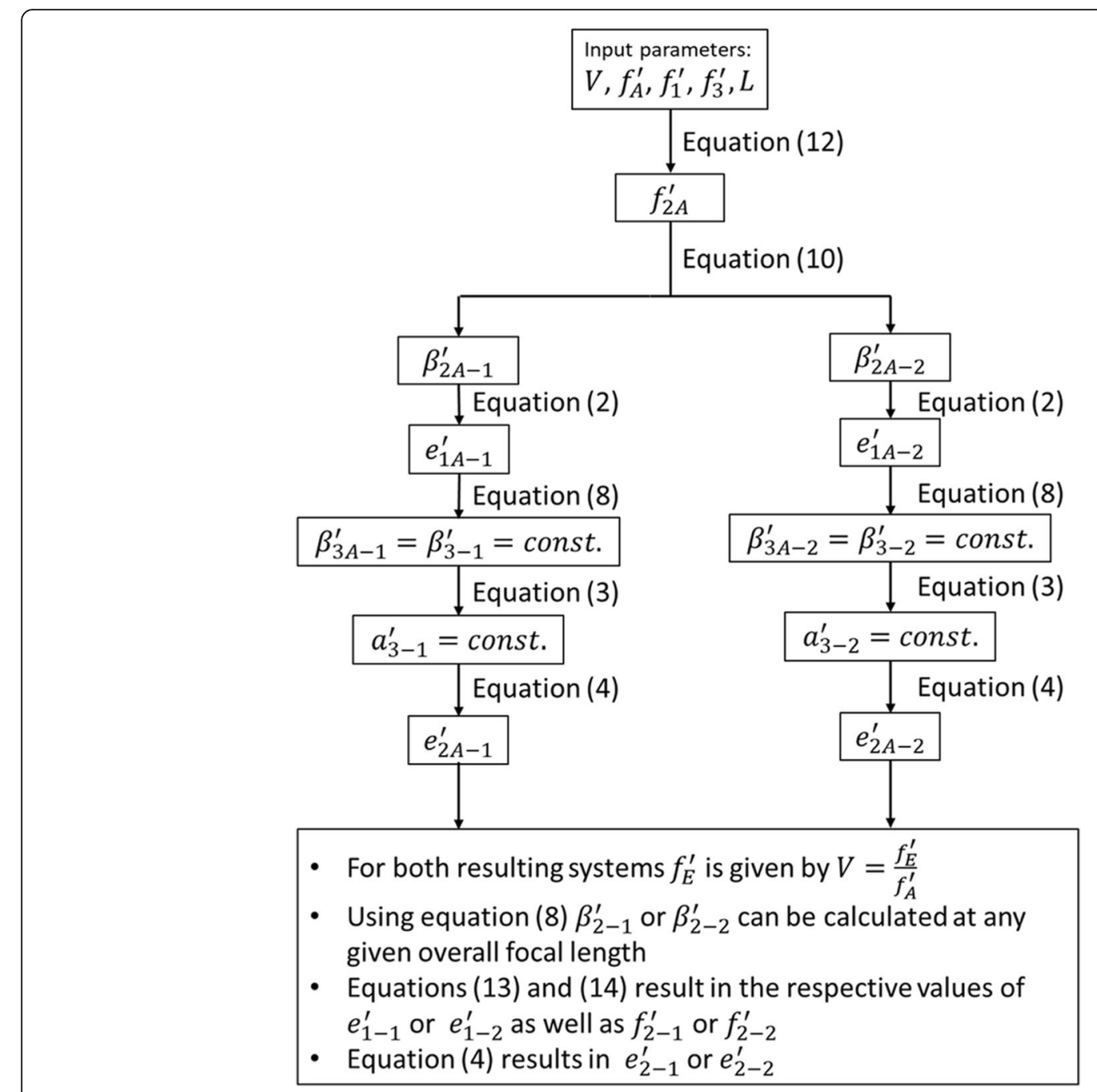

Fig. 3 Sketch of the algorithm for designing hybrid zoom systems

visualize the feasibility of the resulting collinear layouts.

Since the movement within the proposed zoom lens is strictly linear, controlling the partial distances at the beginning and at the end of the zoom range is enough to avoid collisions between the sub-modules. As opposed to this, the focal length of the tuneable sub-module changes nonlinearly, such that the behaviour between the limits of the zoom range has to be considered. This requires controlling a large number of values, which makes the optimization process very time consuming. To avoid this, it is necessary to know the extreme values of the required behaviour of the tuneable sub-module. By doing this we only have to control the limits of the focal range and these extreme values. For this reason, it is worth to have a look at the equations involved:

We can describe the focal length of the tuneable submodule depending on the constant distance between the intermediate image planes one and two, $L_{2}$ :

$$
\begin{aligned}
& L_{2}=a_{2}^{\prime}-a_{2} \\
& a^{\prime}=\frac{a f^{\prime}}{a+f^{\prime}} \\
& f_{2}^{\prime}=a_{2}\left(-1-\frac{a_{2}}{L_{2}}\right)
\end{aligned}
$$

Since $L_{2}$ is constant and $a_{2}$ changes linearly over the zoom range we can use the derivation of (17) to find the extreme values of the focal length variation of the tuneable lens:

$$
\frac{\partial f_{2}^{\prime}}{\partial a_{2}}=-\frac{2}{L_{2}} a_{2}-1
$$

The root of (18) can be found as: 


$$
a_{2}=-\frac{L_{2}}{2}
$$

The second derivation is clearly zero; the sufficient condition of an extremum at (19) is fulfilled.

There are two possibilities to meet condition (19): Firstly, $a_{2}=L_{2}=a_{2}^{\prime}=0$ which would not result in a feasible zoom system and therefore is of no further concern. The second possibility is $a_{2}=-a_{2}^{\prime}$, which means $\beta_{2}^{\prime}=-1$. To receive the value of $f_{2}^{\prime}$ at its extremum $\left(f_{2 E x}^{\prime}\right)$ we can use the simple condition that the distance between object and image for a system with a linear magnification of -1 is four times the system's focal length:

$$
f_{2 E x}^{\prime}=\frac{L_{2}}{4}=\frac{1}{4}\left(L-f_{1}^{\prime}-a_{3}^{\prime}\left(-\frac{1}{\beta_{3}^{\prime}}+1\right)\right)
$$

We now only have to control the values of $f_{2}^{\prime}$ at the beginning and the end of the zoom range, as well as the value at the extremum given by (20), to check if the focal range of our tuneable lens is sufficient over the whole zoom range.

Using these results, we implemented our calculation approach within the software tool Mathematica by Wolfram Research ${ }^{\bullet}$. We set our fixed requirements $V$ and $f_{A}^{\prime}$ as well as the range for the additional parameters $f_{1}^{\prime}, f_{3}^{\prime}$ and $L$. Additionally, we set our requirements for the feasibility like the minimum distance between the sub-modules $e_{\text {min }}^{\prime}$, the minimum focal lengths of the sub-modules $f_{\text {min }}^{\prime}$ and the focal range of the tuneable lens. We can link all the equations that describe our controlled parameters. By using the "RegionPlot3D"- command we can vary the three additional parameters within the set boundaries and plot the region where all our requirements are met. Thereby we get three-dimensional plots of all the feasible collinear layouts within a space defined by the three variable additional parameters $f_{1}^{\prime}, f_{3}^{\prime}$ and $L$ (Fig. 4):

With this approach it is easy to add any additional requirement for the collinear layout. The corresponding system property has to be described by a function depending on the three variable parameters or any other system parameter that can be added to the calculation process. As an example we have already added a condition for the change of the f-number from the beginning to the end of the zoom range. Thereby we only get the solutions which also meet these additional requirements.

\section{Results and discussion}

The illustration of the 3D-solution space obtained by the automated variation of parameters allows us to compare the different regions (Fig. 5). We can use the calculated system properties to analyse the different collinear layouts within the software PARAX [10] developed in-house and decide which region of the solution space is the best for our application. Thereby, we are able to make a targeted choice of a specific zoom lens design and also optimize certain parameters (e.g. the system length $L$ ).

\section{Experimental demonstration}

In order to demonstrate the potential of our method, we have been looking for a collinear layout of a zoom lens suitable for the integration of a specific tuneable lens, available in our lab (APL ${ }^{\mathrm{Tm}}-1050$, Holochip Corp., [11]). Based on measurements we identified the region of the focal length tuning range of this lens where its sensitivity is sufficient to tune the focal length manually. As

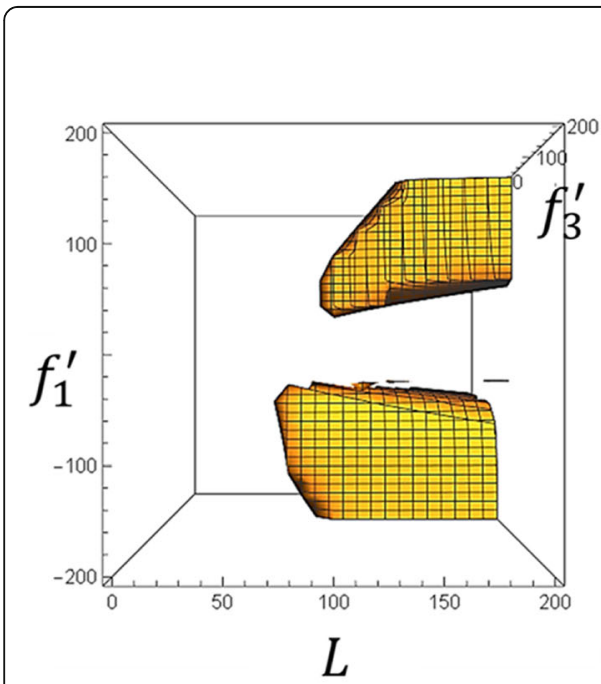

View from above

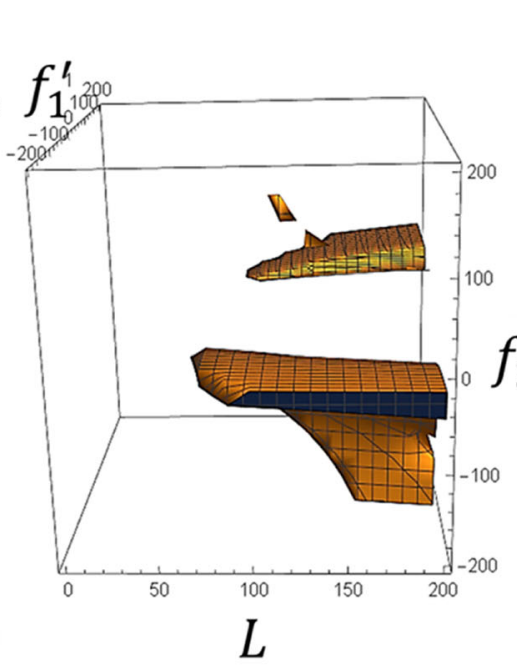

Main view

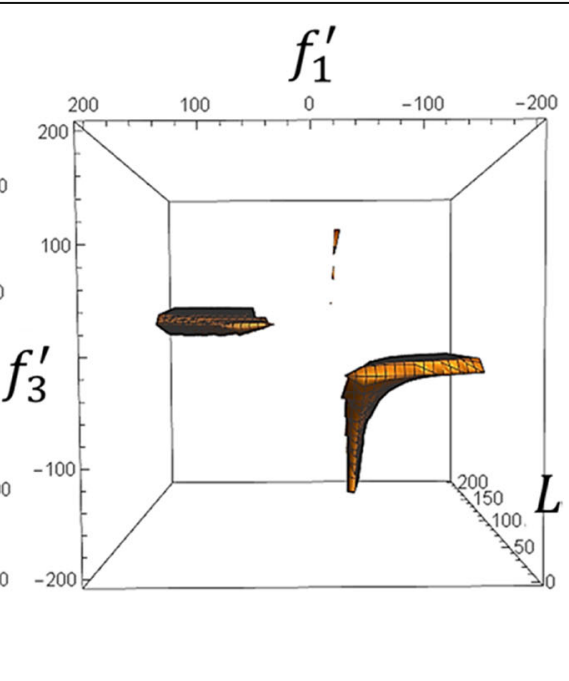

View from the left

Fig. 4 The collinear solution space for the proposed hybrid zoom lens in three viewsRequirements: $\mathrm{V}=3, \mathrm{f}_{\mathrm{A}}^{\prime}=25 \mathrm{~mm}, \mathrm{e}_{\min }^{\prime}=5 \mathrm{~mm}, \mathrm{f}_{\min }^{\prime}=12,5 \mathrm{~mm}$ 


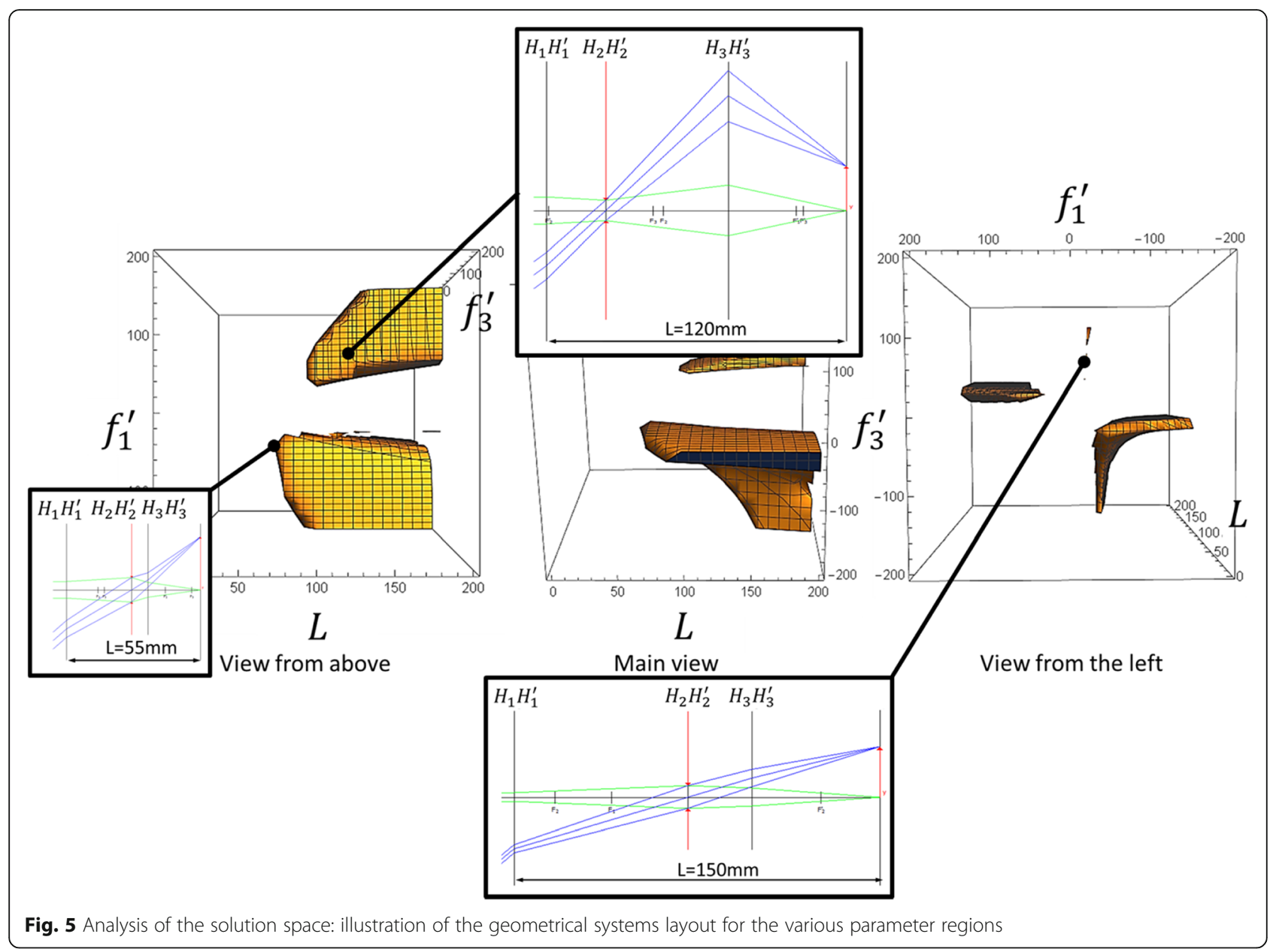

mentioned before, we were able to insert this constraint directly into our automated variation of parameters. In the next step, we made a targeted choice of a system with focal lengths of the fixed submodules that can be realized by lenses available in the lab. Thus the demonstrated experimental setup (Fig. 6, left) shows the feasibility of our proposed 'hybrid zoom lenses'. It also demonstrates the advantage of our targeted calculation approach which allowed us to realize the setup without customized optical elements. The graph of the focal lengths over the zoom range for our experimental system, obtained by using the software PARAX, shows that the change of the overall focal length largely exceeds the required tuning range of the tuneable sub-module (Fig. 6, right). This applies to all examples of the proposed variation of a 'hybrid zoom lens' and shows that there is a potential for realizing zoom ratios far beyond the ratio of $V=3$ shown here. The horizontal axis of the graph is "partial distance 1", meaning the distance between the first and the second sub-module. Since the first sub-module is stationary, this distance directly correlates with the trajectory of the moving second sub- module. So the depicted behaviour of the focal lengths corresponds to the case of a linearly moving second sub-module.

\section{Theoretical considerations and limitations}

Our experimental demonstration system is subject to various limitations. The f-numbers of our system are still fairly high $(9,24-17,99)$. This is mainly due to the small physical aperture of our tuneable lens (of about $10 \mathrm{~mm}$ ). However, this problem seems solvable in the near future since an aperture of about $20 \mathrm{~mm}$ is already enough to achieve more reasonable $\mathrm{f}$ numbers and there are research groups proposing designs for tuneable lenses with diameters of $30 \mathrm{~mm}$ and more (e.g. [12, 13]). Since the intention behind our experimental setup is a demonstration of the general feasibility of our approach we did not focus on a systems optimization with respect to aberrations etc. Instead, we directly assembled our setup according to the collinear layout using simple achromatic- and even singlet lenses. The system can be considered as a demonstrator concerning the necessary focal length change of the tuneable lens as well as its movement 


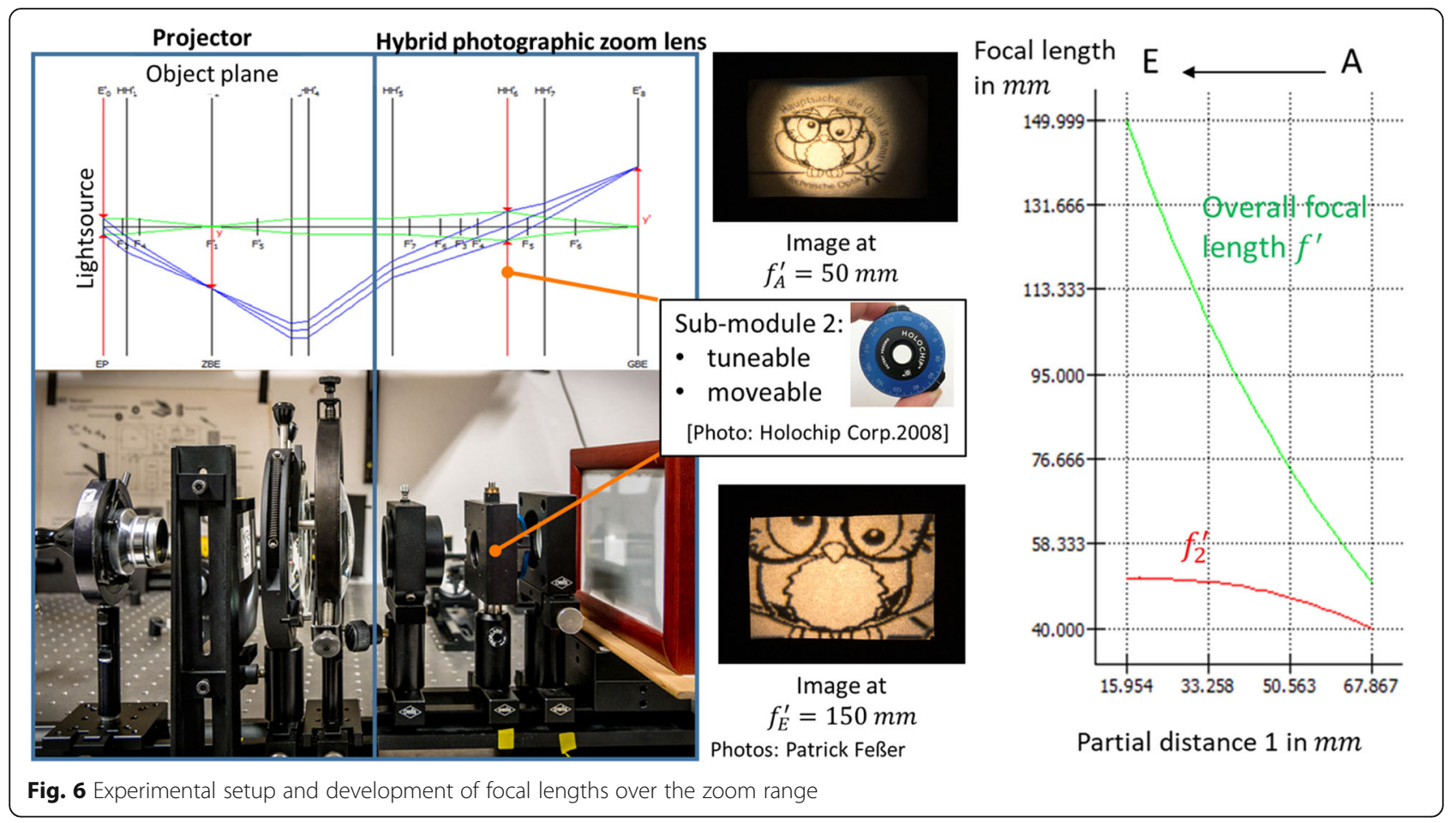

without consideration of any aberrations, which have to be addressed within future work.

We have derived similar approaches for different collinear structures of classical zoom lenses and applied the automated variation of parameters. Similarly, lenses based exclusively on tuneable lenses and a further possible variation of the 'hybrid zoom lens' can be found, which proves the general potential of our approach. To get an impression of the possibilities for reducing the system length by using the hybrid photographic zoom lens we chose some general requirements: A zoom ratio $V=3$, a system focal length at the beginning of the zoom range of $f_{A}^{\prime}=25 \mathrm{~mm}$, a minimum distance between the principal planes of the sub modules $e_{\min }^{\prime}=5 \mathrm{~mm}$ and a minimum focal length for all the sub modules $f_{\min }^{\prime}=12$ , $5 \mathrm{~mm}$. Applying these parameters we search the resulting solution spaces of classical zoom lenses of the mechanically compensated type shown in Fig. 1. Alternatively, the collinear layout of the proposed type of 'hybrid zoom lens' with minimum system lengths is considered. The parameter space is illustrated in the 3D plot before moving to 2D for a more detailed analysis (Fig. 4, Fig. 7).

The same approach was applied to a possible structure of a zoom lens without moving sub-modules, where sub-module 2 and 3 are tuneable. The resulting solution space also offers collinear layouts with system lengths lower than the minimum system length of the classical zoom lenses. As expected, analysing examples of these systems shows far higher requirements for the tuning range of the tuneable lenses than the hybrid zoom lens' layouts. The number of structures considered is quite limited and it must be clear that the collinear system length does not directly translate into the length of the optimized real zoom lens. Nevertheless, the comparisons already show the expected trends: Smaller system lengths can be achieved by the use of tuneable lenses and the requirements on the focal length tuning range can be significantly reduced when comparing the 'hybrid zoom lenses' to the zoom lenses based exclusively on tuneable lenses. Thus further considerations in this field seem promising.

The most significant limitation of the automated variation of parameters at its actual stage is that we can only display the solutions within a space defined by the three additional input parameters. In future work we plan to extend this concept to show the solutions depending on freely chosen system properties.

\section{Conclusions}

We combined the concepts of classical zoom lenses with tuneable lenses with the aim to realize 'hybrid zoom lenses' with a constant image plane and solely linearly moving sub-modules. We presented an approach for systematically finding specific collinear layouts of these systems and have been able to show possible advantages of our variations of zoom lenses. The advantages are the possibility to realize smaller system lengths compared to classical zoom lenses and 


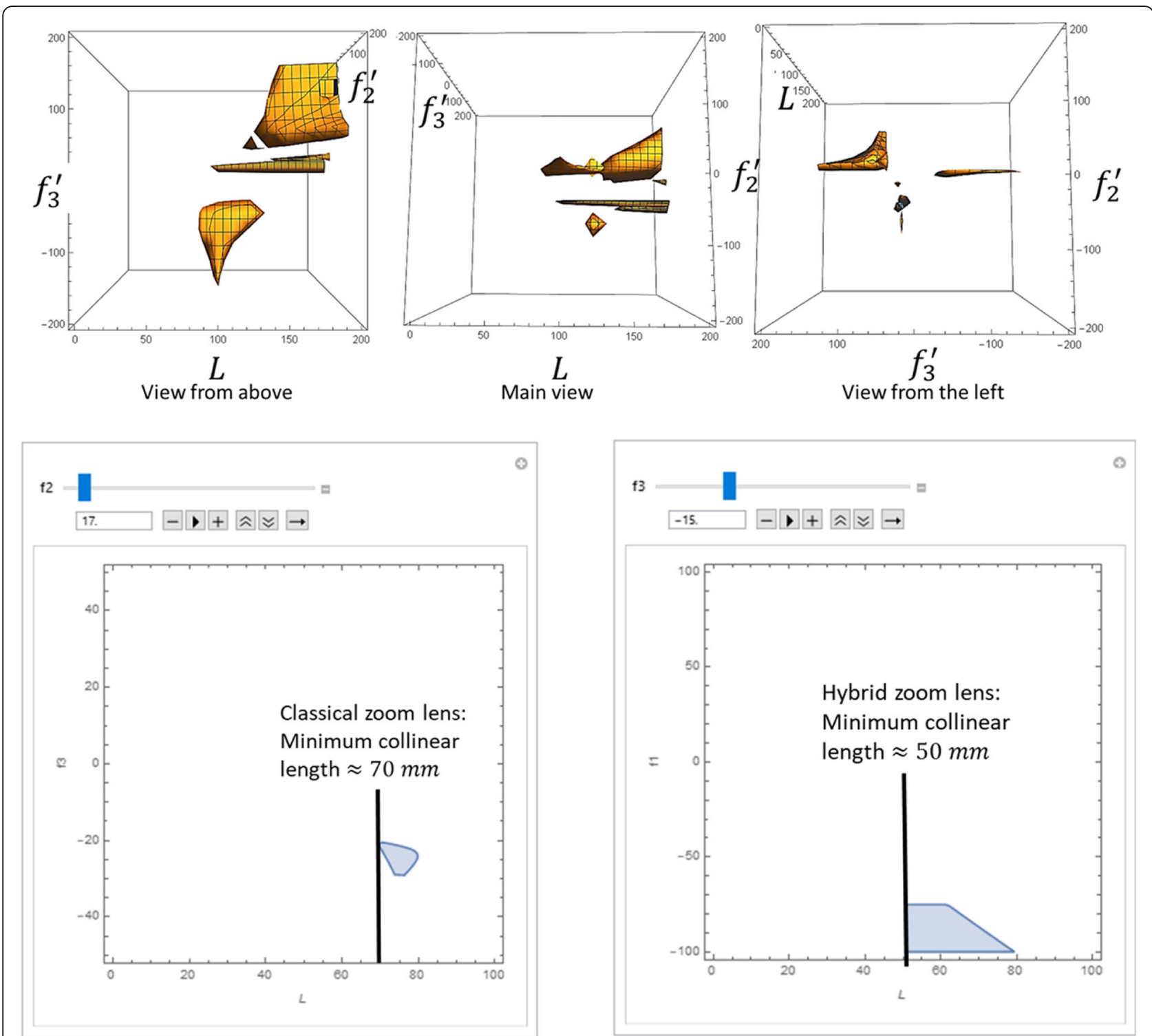

Fig. 7 The collinear solution space for a classical zoom lens of the mechanical compensation type shown in Fig. 1 (above, requirements: $V=3$, $\mathrm{f}_{\mathrm{A}}^{\prime}=25 \mathrm{~mm}, \mathrm{e}_{\min }^{\prime}=5 \mathrm{~mm}, \mathrm{f}_{\min }^{\prime}=12,5 \mathrm{~mm}$ ) and the $2 \mathrm{D}$ analysis of the minimum collinear system lengths for the classical (lower left) and the proposed 'hybrid photographic zoom lens' (lower right)

the reduced requirements on the tuneable lenses focal range [14] [15]. Possible further considerations are to enhance the method of the automated variation of parameters and to analyse additional structures of the 'hybrid zoom lenses' as well as their performance regarding the correction of aberrations.

\section{Acknowledgements}

The authors want to thank all colleagues from the Fachgebiet Technische Optik at TU IImenau for their valuable suggestions and questions. Special thanks go to Mr. Patrick Fesser for taking the photographs of the experimental setup and to Mrs. Katja Richter and Mr. David Fischer for helping with the language of the article.

\section{Authors' contributions}

All authors read and approved the final manuscript.

\section{Funding}

We acknowledge support for the Article Processing Charge by the German Research Foundation (DFG) and the Open Access Publication Fund of the

Technische Universitaet IImenau.

\section{Availability of data and materials}

Not applicable.

Competing interests

The authors declare that they have no competing interests. 
Received: 26 February 2019 Accepted: 16 May 2019

Published online: 07 June 2019

\section{References}

1. Clark, A.D.: Zoom Lenses. Adam Hilger LTD, London (1973)

2. Leopold, S., Polster, T., Pätz, D., Knöbber, F., Ambacher, O., Sinzinger, S. Hoffmann, M.: MOEMS tunable microlens made of aluminum nitride membranes. J. Micro/Nanolith. MEMS MOEMS. 12(2), 023012 (2013) https:// doi.org/10.1117/1.JMM.12.2.023012

3. Stürmer, M., Wapler, M., Vogel, G.: Piezoaktor macht Flachglas zur variablen Linse. Photonik, vol. 48, pp. 38-39 (2016)

4. Bernet, S., Ritsch-Marte, M.: Adjustable refractive power from diffractive Moiré elements. Appl. Opt. 47, 3722-3730 (2008) https://doi.org/10.1364/ AO.47.003722

5. Grewe, A., Fesser, P., Sinzinger, S.: Diffractive array optics tuned by rotation. Appl. Opt. 56, A89-A96 (2017) https://doi.org/10.1364/AO.56.000A89

6. Pätz, D., Sinzinger, S., Leopold, S., Hoffmann, M.: 3D Scanning system based on tunable cylindrical lenses. In: DGaO Proceedings 113.Tagung, Eindhoven, Netherlands (29 May-2 June 2012)

7. Pätz, D., Leopold, S., Hoffmann, M., Sinzinger, S.: Tunable anamorphotic imaging system based on fluidic cylindrical lenses, pp. 17-21. Paper presented at the international conference on optical MEMS and Nanophotonics, Glasgow, Scotland (August 2014)

8. Yedid, I: The evolution of zoom camera technologies in smartphones. Corephotonics. http://corephotonics.com/industry-news/evolution-zoomcamera-smartphones. Accessed 18 Feb 2019 (2017).

9. Aschwanden, M, Bueeler, M, Salt, M for: OPTOTUNE AG: Optical zoom lens with two liquid lenses. International Patent WO 2015/024136 A1, 26. Feb 2015

10. Mitschunas, B., Rudolf, B., Bielert, R., Mitschunas, J., Sinzinger, S.: Kollineare Modellierung komplexer optischer Systeme. Photonik. 48, 46-49 (2016)

11. Holochip Corporation: APL-1050 Product Brochure. https://docs.wixstatic. com/ugd/1b241a_82f78499327e42b991a76ee611dc824d.pdf (2008). Accessed 1 May 2018

12. Nazmul, H., Hanseup, K., Mastrangelo, C.H.: Large aperture tunable-focus liquid lens using shape memory alloy spring. Opt. Express. 24(12), 1333413342 (2016) https://doi.org/10.1364/OE.24.013334

13. Wang, L., Oku, H., Ishikawa, M.: Variable-focus lens with $30 \mathrm{~mm}$ optical aperture based on liquid-membrane-liquid structure. Appl. Phys. Lett. 102, 131111 (2013) https://doi.org/10.1063/1.4800603

14. Lenk, L., Mitschunas, B., Sinzinger, S.: Zoomoptiken mit verstimmbarer Optik und linearer Verschiebung der Einzelmodule. In: DGaO Proceedings 119. Tagung, Aalen, Germany, 22-26 (May 2018)

15. Lenk, L., Mitschunas, B., Sinzinger, S.: Zoom Lenses with Tunable Lenses and Linear Lens Movements. Paper presented at EOSAM 2018, Delft, Netherlands (8-12 October 2018)

\section{Publisher's Note}

Springer Nature remains neutral with regard to jurisdictional claims in published maps and institutional affiliations.

\section{Submit your manuscript to a SpringerOpen ${ }^{\circ}$ journal and benefit from:}

- Convenient online submission

- Rigorous peer review

- Open access: articles freely available online

- High visibility within the field

- Retaining the copyright to your article

Submit your next manuscript at $\boldsymbol{\nabla}$ springeropen.com 